\title{
Imaging septaria geobody in the Boom Clay using a Q-compensated reverse-time migration
}

\author{
J.M. Carcione ${ }^{1, *}$, T. $\mathrm{Zhu}^{2,3}$, S. Picotti ${ }^{1} \&$ D. Gei ${ }^{1}$
}

1 Istituto Nazionale di Oceanografia e di Geofisica Sperimentale (OGS), Borgo Grotta Gigante 42c, 34010 Sgonico, Trieste, Italy

2 Jackson School of Geosciences, University of Texas at Austin, Austin, TX 78758, USA

3 Department of Geosciences and Institute of Natural Gas Research, The Pennsylvania State University, University Park, PA 16802, USA

* Corresponding author. Email: jcarcione@inogs.it

Manuscript received: 07 0ctober 2015, accepted: 20 December 2015

\section{Abstract}

The Boom Clay is being investigated as a host rock for disposal purposes of radioactive wastes. Although the formation is relatively uniform and homogeneous, there are embedded septaria bodies (carbonates) or layers of septaria that may constitute a problem regarding the integrity of the clay. It is therefore essential to locate these geobodies, particularly with seismic experiments. Since the medium shows strong attenuation it is necessary to correct for this amplitude loss if true amplitudes of the reflections are required when imaging these bodies after the stack. To achieve this task, we implement a reverse-time migration algorithm based on a dispersionless anelastic rheology, that is, the phase velocity and attenuation factor are frequency independent, and back-propagation is performed with a negative quality factor, $Q$. The algorithm is tested on synthetic data. For this we assume that the septaria are diffractors generating waves synchronously to simulate a stacked seismic section, that is, the result of an exploding-reflector experiment. In this case, back-propagation is stopped when all the diffraction points are imaged at the same time. The examples consider layers of septaria and isolated septaria embedded in homogeneous and inhomogeneous Boom Clay with zones of low $Q$. The amplitude of the geobodies is recovered and the resolution is improved, even in the presence of noise.

Keywords: Boom Clay, septaria, seismic migration

\section{Introduction}

The Boom Clay in Belgium is composed of clays and generally has embedded boulders and/or thin layers of septaria (according to the Merriam Webster dictionary, a septarium is 'a concretionary nodule usually of limestone or clay ironstone intersected within by cracks filled with calcite, barite, or other minerals'). The formation of these concretions (lithification) started early in the diagenesis process near the sediment-water interface and before compaction occurred (De Craen et al., 2004). The septaria generally are made of ellipsoidal-shaped carbonate concretions, whose sizes range from a few decimetres up to a couple of metres (see Fig. 1). The seismic response of these thin layers is characterised by a set of parallel reflection events, which, depending on the frequency bandwidth, look like continuous horizontal events or alignments of diffraction events (Fig. 2) (see Hemerijckx et al., 1983; his Fig. 2).

The septaria may constitute a problem regarding the integrity of the Boom Clay and therefore it is essential to locate these geobodies, preferably with seismic waves. However, the fact is that the presence of high attenuation often degrades the seismic images, resulting in a low resolution of targets. In this case the conventional migration may not image the geobodies properly.

Recently, this problem has been reconciled with an advanced migration with attenuation compensation. For example, Wang \& Guo (2004) presented a migration algorithm incorporating inverse $Q$ filtering to improve the imaging resolution, although the imaging technique is limited to $1 \mathrm{D}$ velocity and attenuation models. Zhang et al. (2010) proposed a reverse-time migration 


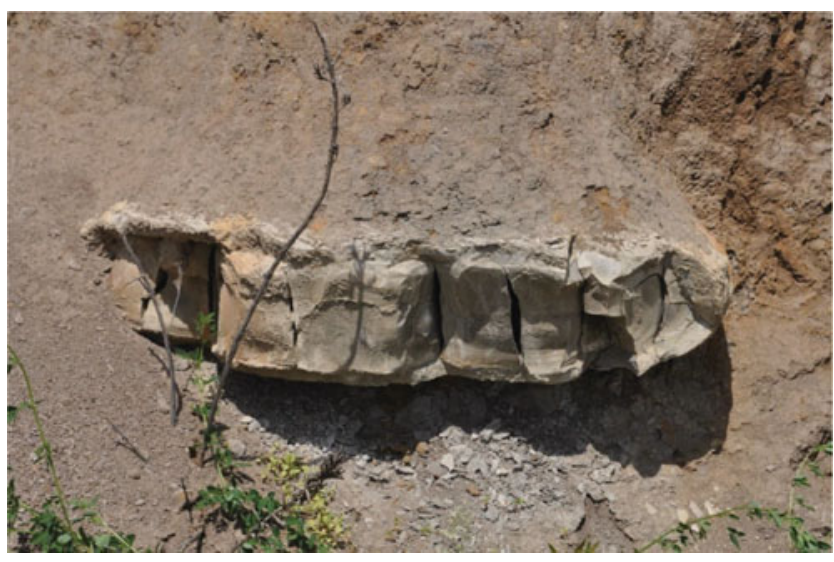

Fig. 1. A calcareous septarium embedded in Boom Clay. The width is approximately $60 \mathrm{~cm}$ (from Vis \& Verweij, 2014).
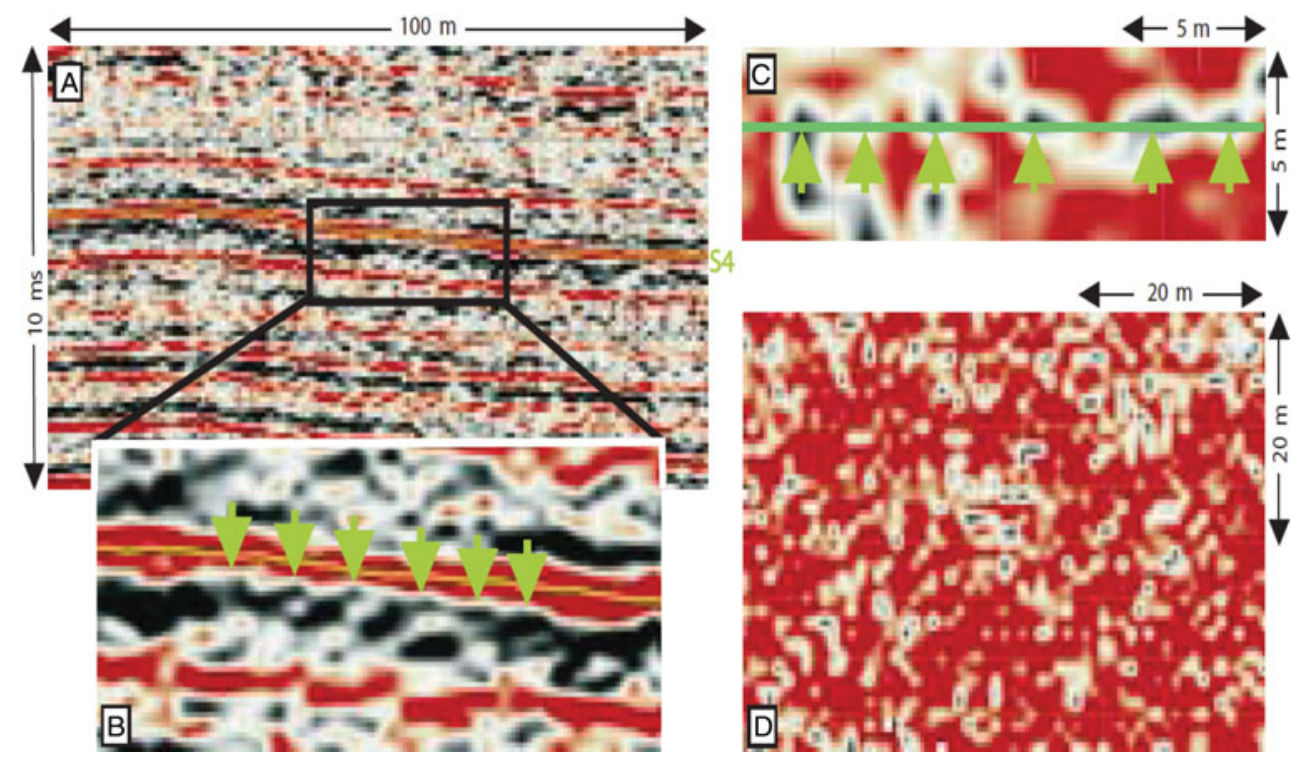

Fig. 2. A. In-line section showing a septaria level; B. Close-up with single diffraction events; $C$ Horizon slice at 0.2 ms below that level; the green arrows refer to events identified in B; D. Enlarged section showing typical blotchy pattern possibly suggesting concretion distribution (from Missiaen et al., 2002).

(RTM) scheme for compensating attenuation and phase dispersion effects. Their viscoacoustic wave equation is based on a constant- $Q$ model, that is, attenuation is considered to be approximately linear with frequency. Dutta \& Schuster (2014) used a linearized 2D viscoacoustic wave equation based on the Zener model, written in the particle velocity-stress formulation. The method is adapted from conventional least-squares migration and reconstructs the Earth's reflectivity image from the recorded wavefield under the Born approximation. Zhu (2014) implemented a time-reverse modelling approach (or reverse-time focusing) based on a viscoacoustic wave equation which explicitly separates attenuation and dispersion following a constant- $Q$ model based on fractional derivatives (Carcione, 2010). Later this idea was formulated in an RTM algorithm (Zhu et al., 2014). Because of possible instability problems, attenuation compensation is necessary to stabilise the migration.
Sena et al. (2006) introduced a plane-wave approximation in the split-step Fourier technique in the frequency domain to stabilise the algorithm in the presence of attenuation. Zhu et al. (2014) and Sun et al. (2015) adapted a low-pass filter to suppress the high-frequency noise during the compensation.

In this work, we propose to use a post-stack RTM (e.g. Baysal et al., 1983) taking into account the attenuation effects to recover the migration amplitude of septaria geobodies. We use the simple exploding-reflector concept to back-propagate the seismic waves. To extrapolate source and receiver wavefields with attenuation compensation, the equation used for backpropagation of the signals has the same velocity but a negative $Q$ factor. We solve a lossy wave equation that is dispersionless. In the migration process, the recorded seismogram is used as a time-dependent boundary condition. The seismic trace is applied at each receiver in reversed time and the propagation 
Fig. 3. Septaria bodies and layers embedded in homogeneous Boom Clay. The upper layer is composed of septaria bodies of $1 \mathrm{~m}$ size separated with a period of $4 \mathrm{~m}$. The period is $10 \mathrm{~m}$ for the lower layer. Moreover, there are isolated septaria at different depths. The $\mathrm{Q}$ factor of the Boom Clay at the left-hand side is 20 (surrounded by a box with dashed grey lines), while the right-hand zone is lossless.

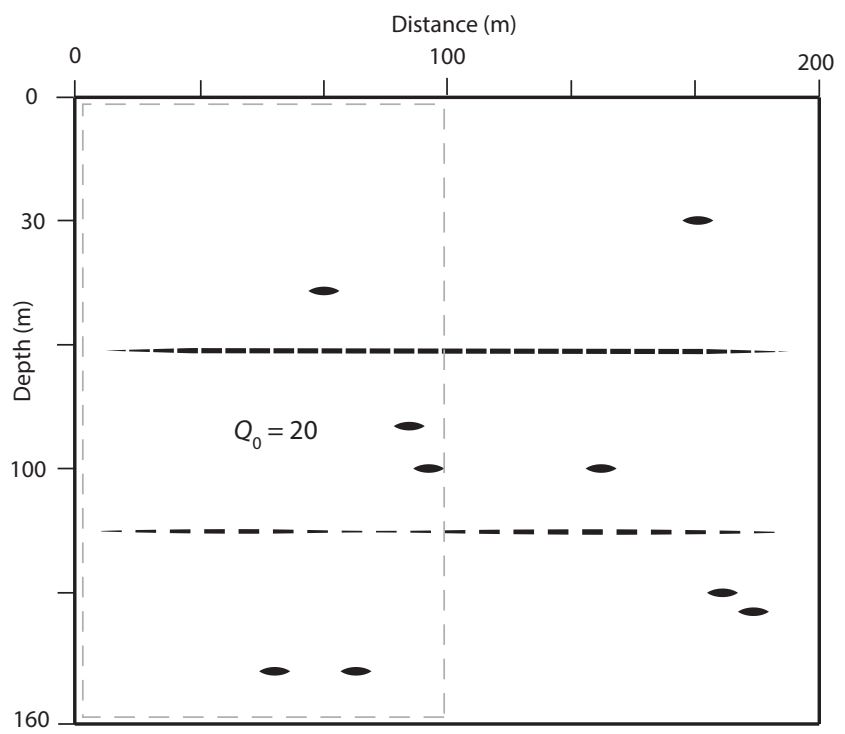

Distance $(\mathrm{m})$

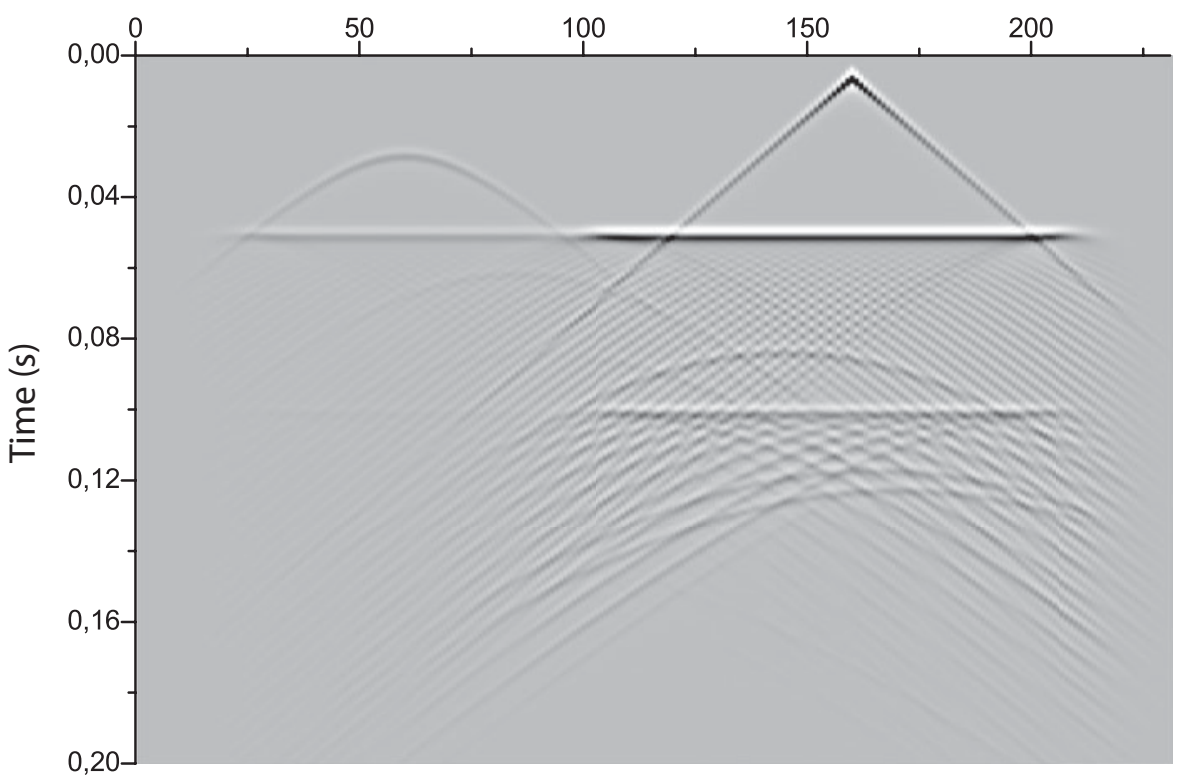

goes back in time until the origin time, where the best focusing occurs. The reflectors are thus imaged. We also apply a low-pass filter to suppress the high-frequency noise.

\section{The dispersionless wave equation}

Wave motion ( $\mathrm{P}$ waves) in the $(x, z)$ plane of an acoustic medium is governed by the following equation:

$$
\partial_{x}\left(\rho^{-1} \partial_{x} p\right)+\partial_{z}\left(\rho^{-1} \partial_{z} p\right)=\left(\rho c^{2}\right)^{-1} \partial_{t t} p+s(x, z, t)
$$

(e.g. Carcione 2015, Chapter 9), where $p$ is the pressure field, $\rho$ is the mass density, $c$ is the wave velocity, $s$ is a source term, $\partial_{i}$ is the partial derivative with respect to the spatial variable $x_{i}\left(x\right.$ or $z$ ) and $\partial_{t t}$ is the second-order partial derivative with respect to the time variable $t$. This equation can be rewritten as

$$
\partial_{t}\left(\begin{array}{c}
p \\
\dot{p}
\end{array}\right)=\left(\begin{array}{cc}
-\gamma & 1 \\
\rho c^{2}\left[\partial_{x}\left(\rho^{-1} \partial_{x}\right)+\partial_{z}\left(\rho^{-1} \partial_{z}\right)\right] & -\gamma
\end{array}\right)\left(\begin{array}{l}
p \\
\dot{p}
\end{array}\right)+\left(\begin{array}{c}
0 \\
s^{\prime}
\end{array}\right),
$$

where $\dot{p}=\partial_{t} p$ (if $\gamma=0$ ), $s^{\prime}=\rho c^{2} s$ and $\gamma=0$ yields equation (1). The introduction of $\gamma$ implies wave attenuation, as we shall see below.

Eliminating the variable $\dot{p}$ in equation (2), we obtain

$\partial_{t t} p=\rho c^{2}\left[\partial_{x}\left(\rho^{-1} \partial_{x} p\right)+\partial_{z}\left(\rho^{-1} \partial_{z} p\right)\right]-2 \gamma \partial_{t} p-\gamma^{2} p+s^{\prime}$. 
A Distance $(\mathrm{m})$
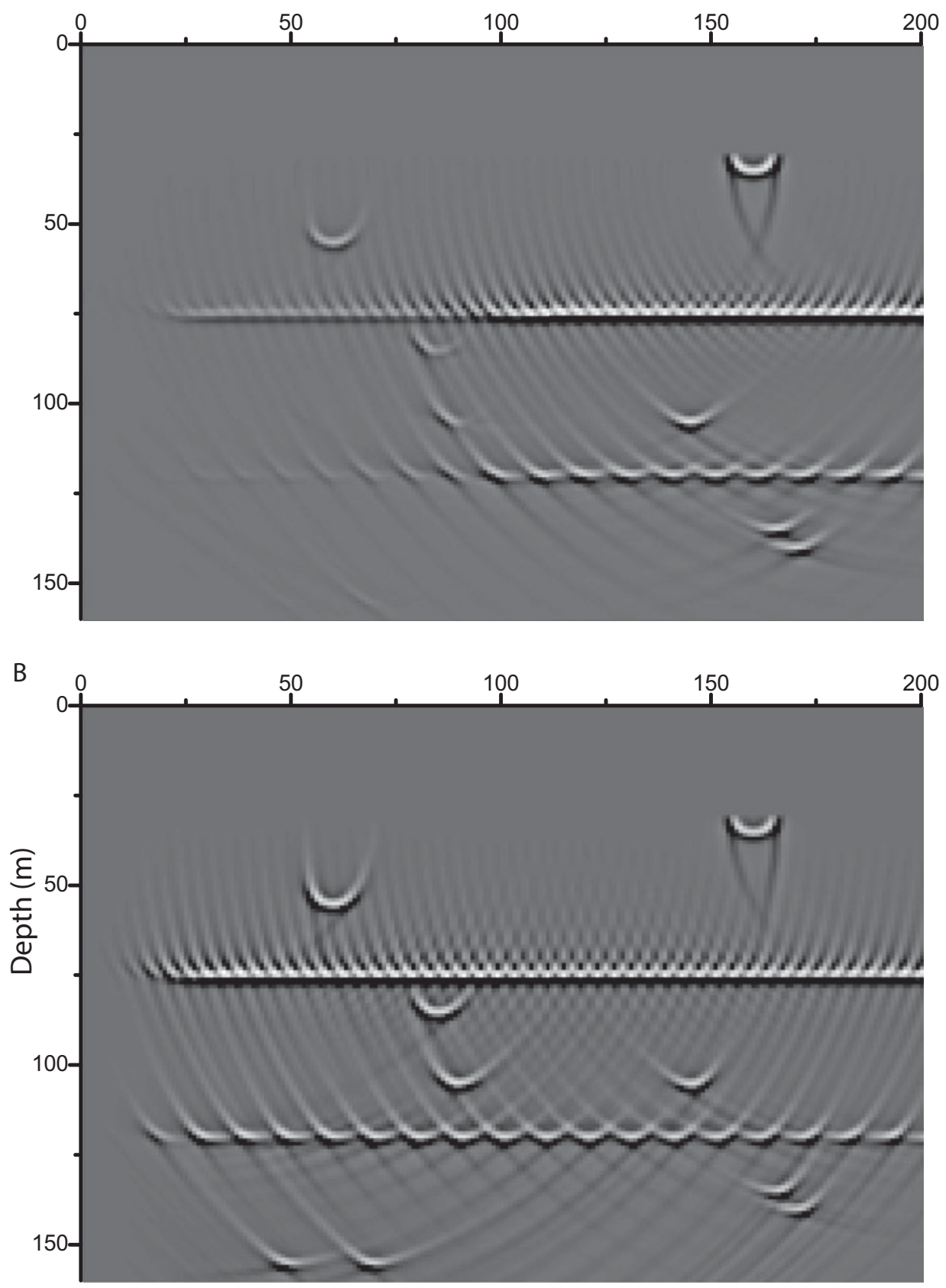

Fig. 5. Imaging by RTM without (A) and with (B) Q compensation, corresponding to the data shown in Fig. 4.

We analyse the anelastic properties related to equation (3) in Appendix A. As shown here, a negative quality factor (implicit in equation (3)) is required to back propagate the wavefield for recovering the amplitudes in reverse-time imaging ( $\mathrm{Zhu}, 2014)$. Thus, the reverse-time imaging equation is written as

$$
\partial_{t t} p=\rho c^{2}\left[\partial_{x}\left(\rho^{-1} \partial_{x} p\right)+\partial_{z}\left(\rho^{-1} \partial_{z} p\right)\right]+2 \gamma \partial_{t} p-\gamma^{2} p+s^{\prime} .
$$

In Appendix B we derive finite-difference versions of these equations to perform forward and backward propagation.
An exploding-reflector version of equation (3) can be obtained by assuming the impedance $\rho c$ constant in the whole space. In this way, multiples are avoided. An approximation to a stacked section is obtained in a single experiment by halving the velocities and by initiating the sources at time zero on all the reflecting boundaries. With this model, the recorded surface time section approximates the stacked or zero-offset section (Carcione et al., 1994, 2002). In a forward simulation, the strength of each source at the interfaces is proportional to the normal-incidence reflection coefficient. Equation (3), for the exploding-reflector approach, 


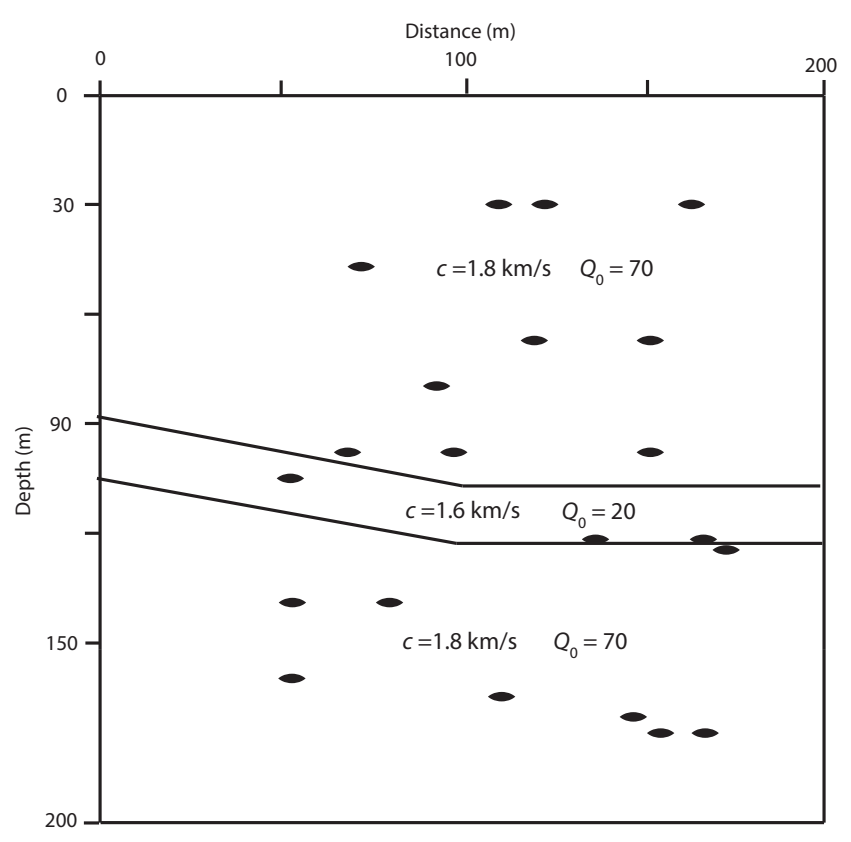

A

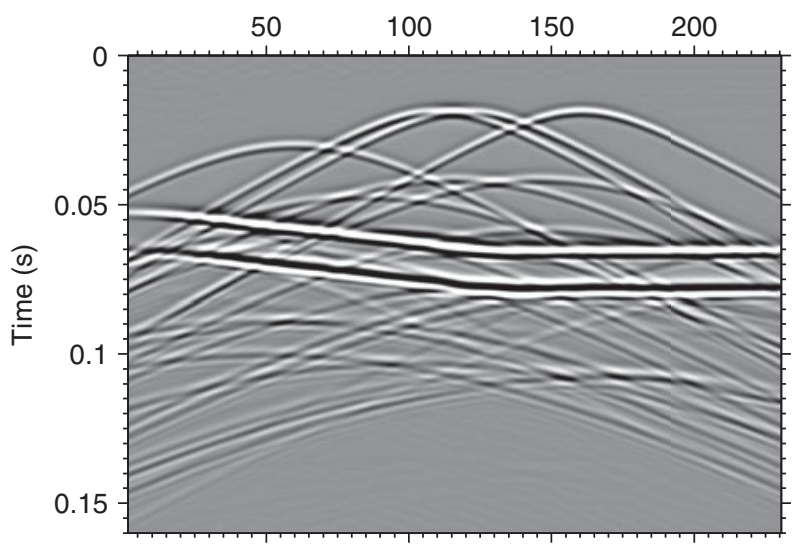

C

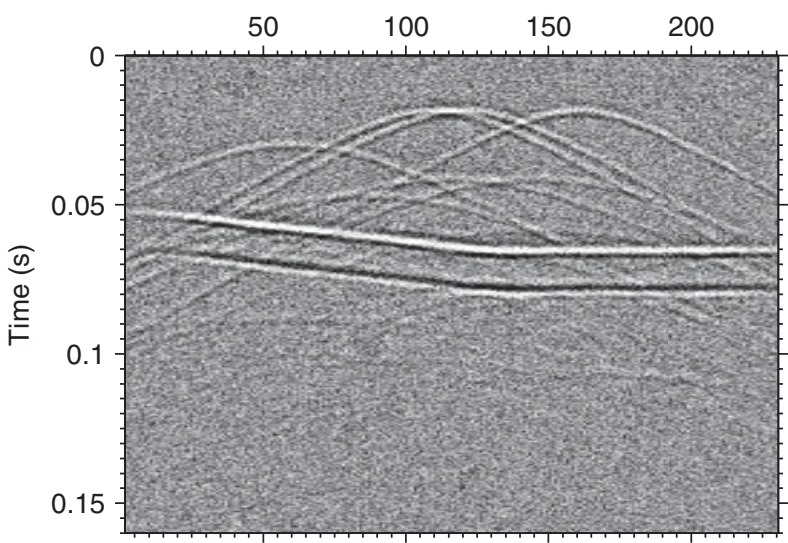

B

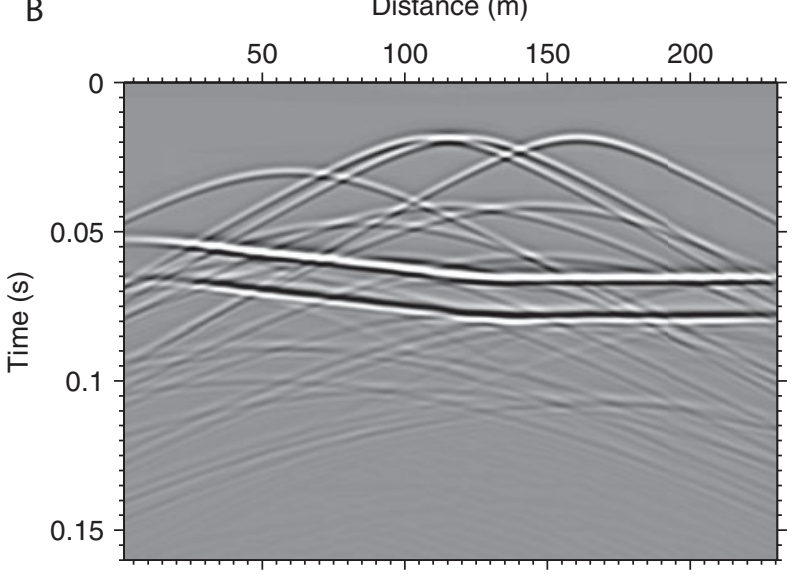

D

Distance $(\mathrm{m})$

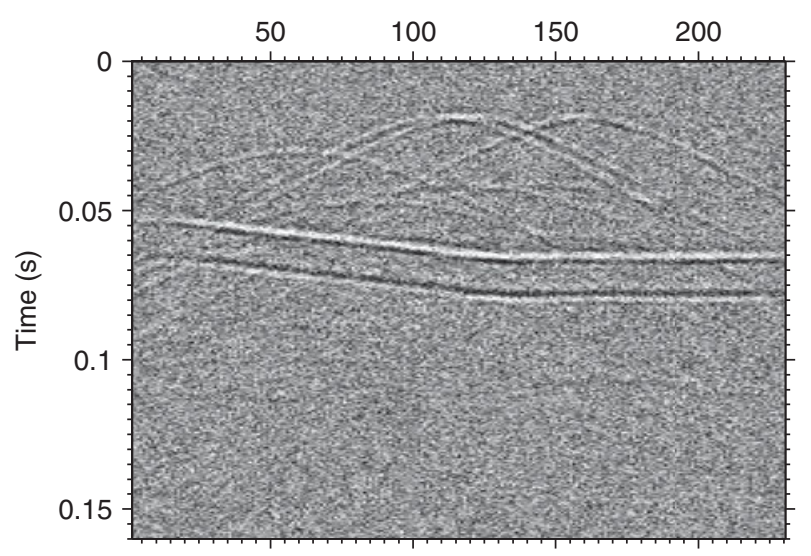

Fig. 7. Synthetic seismograms computed with the exploding-reflector method, corresponding to the model shown in Fig. 6. A. Lossless; B. Lossy; C. Lossy with noise $(S / N=10 \mathrm{~dB}) ; D$. Lossy with noise $(S / N=5 \mathrm{~dB})$. 
A

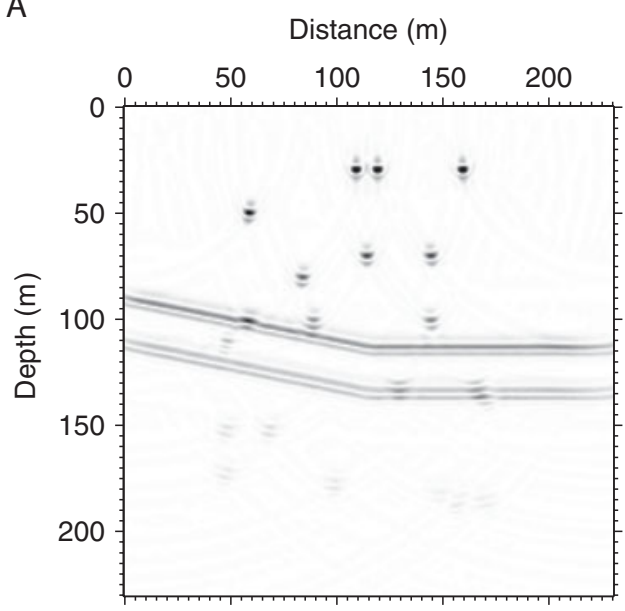

B

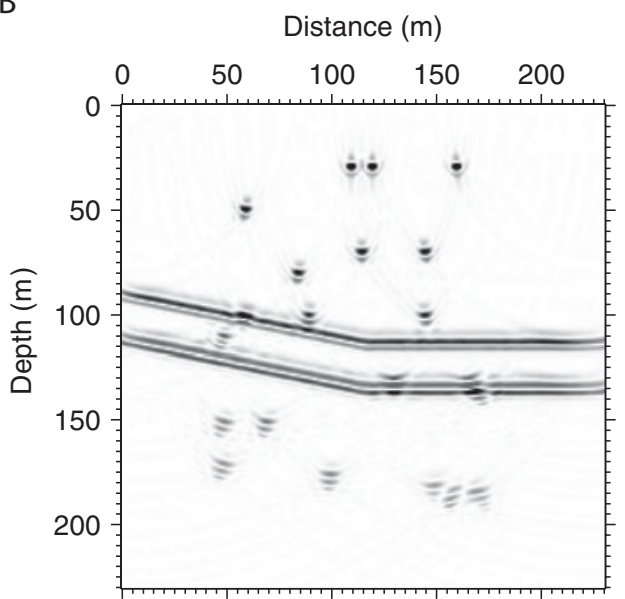

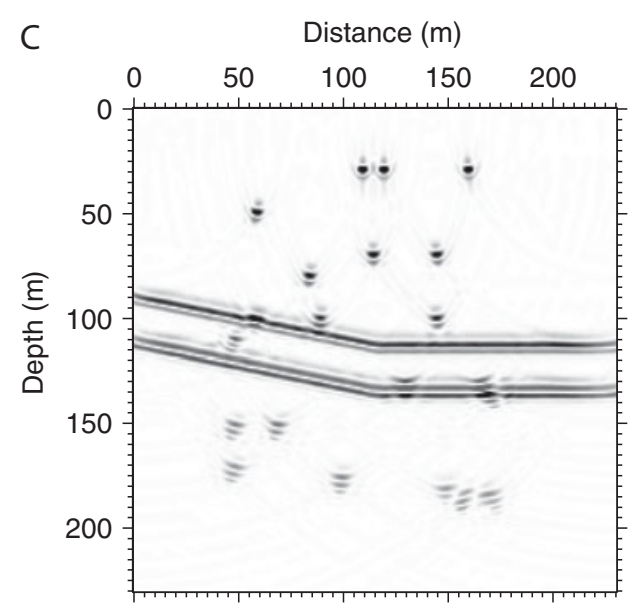

Fig. 8. RTM of the seismograms shown in Fig. $7 B$, without ( $A$ ) and with (B) Q compensation; C. RTM of the seismogram shown in Fig. 7a (lossless case).

becomes

$$
\partial_{t t} p=c\left[\partial_{x}\left(c \partial_{x} p\right)+\partial_{z}\left(c \partial_{z} p\right)\right]-2 \gamma \partial_{t} p-\gamma^{2} p+s^{\prime}
$$

\section{Examples}

First, we consider the model displayed in Fig. 3, where septaria layers and boulders are embedded in homogeneous Boom Clay. The upper thin layer is composed of septaria bodies of $1 \mathrm{~m}$ size separated with a period of $4 \mathrm{~m}$. The period is $10 \mathrm{~m}$ for the lower layer. Moreover, there are isolated septaria at different depths. The seismic velocity of the Boom Clay (homogeneous) is $c=$ $1.8 \mathrm{~km} / \mathrm{s}$ and the attenuation parameter is $Q_{0}=20$ on the lefthand side of the model $(x<100 \mathrm{~m})$ and $Q_{0}=\infty$ on the righthand side. We perform an exploding-reflector simulation based on equation (4) using a Ricker wavelet with a peak frequency $f_{p}=200 \mathrm{~Hz}$. The waveform is

$$
w(t)=\left(u-\frac{1}{2}\right) \exp (-u), \quad u=\left[\frac{\pi\left(t-t_{s}\right)}{T}\right]^{2}
$$

where $T=1 / f_{p}$ is the period of the wave and we take $t_{s}=$ $1.4 T$.

We consider a numerical mesh with $n_{x}=n_{z}=231$ grid points and a grid spacing $d x=d z=1 \mathrm{~m}$. Absorbing boundaries of size 24 grid points are implemented at the sides of the mesh. The algorithm for forward modelling, based on the Fourier pseudospectral method, uses a time step of $0.1 \mathrm{~ms}$ to propagate the wavefield 2000 steps. Fig. 4 shows the synthetic seismogram, where it is clear that the signal has been highly attenuated on the left-hand side. On the right-hand side the response of the layered sets of septaria is continuous and single geobodies cannot be distinguished. This is a Fresnel-zone effect (see Appendix C). According to the equations reported in Appendix $C$ and the properties used here, the Fresnel radii before and after migration at the peak frequency are $R=2.12 \sqrt{z}$ and $R=2.25 \mathrm{~m}$, respectively. The first and second layers of septaria have $z=$ $70 \mathrm{~m}$ and $115 \mathrm{~m}$, implying that before migration the Fresnel radii are $18 \mathrm{~m}$ and $23 \mathrm{~m}$, respectively. The geobodies therefore cannot be discriminated in the unmigrated section, since their separations are 4 and $10 \mathrm{~m}$, respectively. The migration without 
(a) and with (b) $Q$ compensation is displayed in Fig. 5, where it is clear that the amplitude has been recovered (the narrow left vertical zone is within the absorbing boundary, implemented to avoid wraparound). Moreover, the imaging collapses the energy such that the single septaria in the layers can be discriminated, since the horizontal Fresnel radius after migration is $2.25 \mathrm{~m}$, as we have seen above. The two septaria at the left-hand side at a depth of $150 \mathrm{~m}$ have been recovered, even if the seismic responses of these geobodies cannot be seen in Fig. 4. A gain compensation could in principle recover amplitudes, but for events whose signal-to-noise ratio is high, since this method enhances the noise and the signal. On the other hand, $Q$ compensation enhances the signal compared to the noise. Examples with noise are given at the end of this section.

Fig. 6 shows a model where the medium is inhomogeneous, with a high-loss layer embedded in the Boom Clay. The septaria geobodies are denoted by black-diamond shapes. The simulation parameters are the same as in the previous example. The Ricker wavelet is used as a source, with a peak frequency of $200 \mathrm{~Hz}$, and the time step is $0.08 \mathrm{~ms}$. The synthetic seismograms are displayed in Fig. 7, where it can be observed that the septaria below the layer are attenuated with respect to those above the layer (Fig. 7B). The migration without (a) and with (b) $Q$ compensation is displayed in Fig. 8. It can be seen that the septaria geobodies located below the high-loss layer are not well imaged in Fig. 8A, while they have been recovered using compensated RTM imaging in Figure 8B. Compared to the reference image in Fig. 8C, all the diffractors are clearly delineated.

In order to validate the robustness of the algorithm with random noise, we corrupted the seismograms with strong random noise (see Figs 7C and 7D). The diffractions from septaria bodies are not easy to identify. During compensation, a lowpass Tukey filter with a cut-off frequency of $350 \mathrm{~Hz}$ and taper ratio 0.1 is applied. The cut-off frequency has been determined by identifying the noise level in the seismic data spectrum. The imaging results are presented in Figs 9A and 9B. The background appears noisy but both migrated sections clearly show the septaria geobodies.

\section{Conclusions}

Conventional seismic processing is designed to restore amplitudes by a time-dependent scaling, but this procedure introduces amplitude and phase errors to the reconstruction of reflectors since it is not based on the geological model. Moreover, this simple scaling cannot recover the correct location of the interfaces, therefore seismic migration with loss compensation is the correct method to recover a true amplitude wavefield. Here, we have presented a seismic migration methodology to compensate for attenuation loss effects in the post-stacked domain. The approach is based on the exploding-reflector concept, where back-propagation is performed by taking a negative
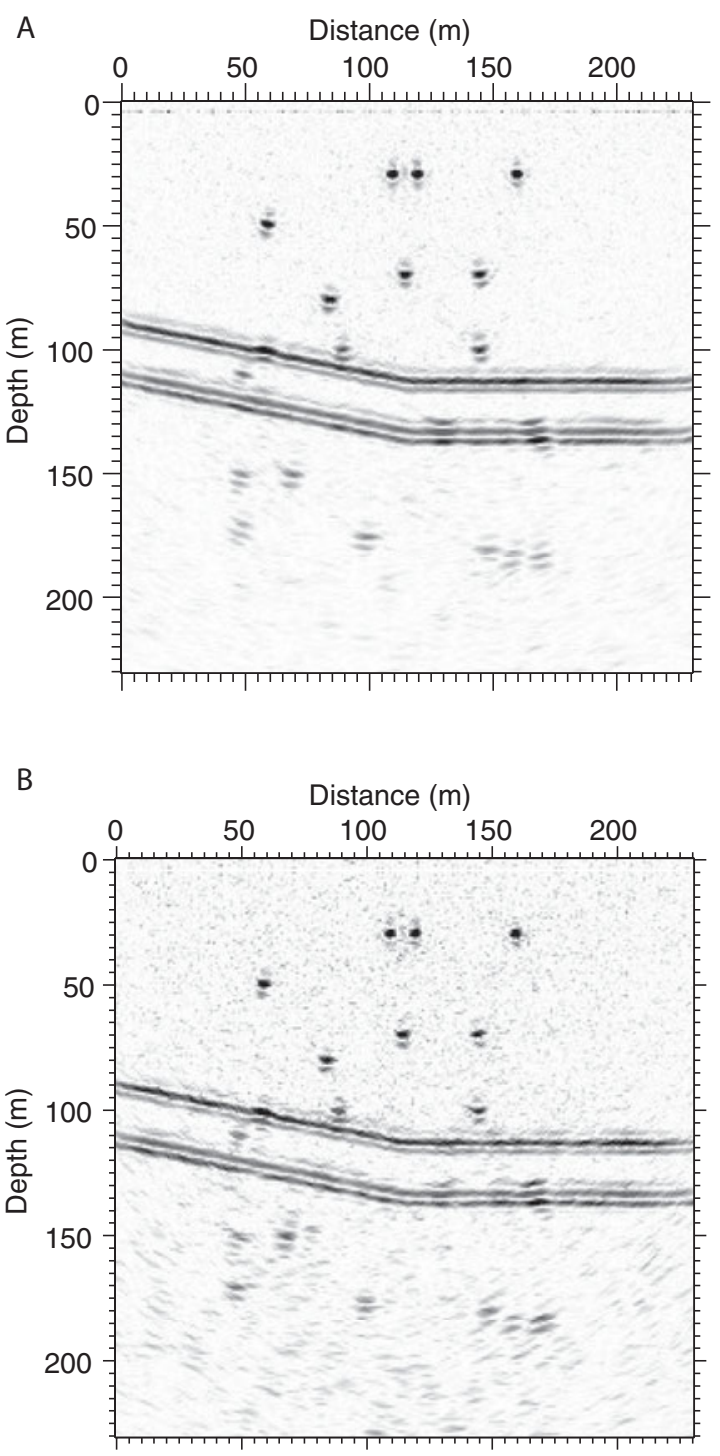

Fig. 9. RTM corresponding to the seismograms shown in Figs $7 C(A)$ and $7 D(B)$.

quality factor. The viscoacoustic equation used for the propagation is dispersionless, that is, the phase velocity is frequencyindependent.

The imaging method was applied to image septaria bodies and thin layers present in the Boom Clay in Belgium. The first example considers a homogeneous medium in terms of velocity but inhomogeneous regarding the attenuation properties. The amplitudes of the septaria embedded in a high-loss zone are fully recovered and an analysis based on the Fresnel-zone concept indicates that the resolution is greatly improved after migration. The second example considers a fully inhomogeneous medium and isolated septaria at random locations. It can be seen that the amplitudes of the septaria geobodies located below a high-loss thick layer have been recovered and the geobodies correctly imaged, even in the presence of different levels of noise. 
The results indicate that this approach can effectively improve the resolution and quality of images, particularly at and beneath high-attenuation zones.

\section{Acknowledgments}

T. Zhu is grateful to the Jackson Postdoctoral Fellowship at the University of Texas at Austin for his financial support.

\section{References}

Abramowitz, M. \& Stegun, I.A. (eds), 1964. Handbook of mathematical functions. Applied Mathematical Series. National Bureau of Standards (Washington, DC).

Baysal, E., Kosloff, D. \& Sherwood, J.W.C., 1983. Reverse-time migration. Geophysics 48: 1514-1524.

Carcione, J.M., 2010. A generalization of the Fourier pseudospectral method. Geophysics 75: 53-56.

Carcione, J.M., 2015. Wave Fields in Real Media. Theory and numerical simulation of wave propagation in anisotropic, anelastic, porous and electromagnetic media, 3rd edition. Elsevier (Amsterdam).

Carcione, J.M., Böhm, G. \& Marchetti, A., 1994. Simulation of a CMP seismic section. Journal of Seismic Exploration 3: 381-396.

Carcione, J.M., Feliciangeli, Piñero, L. \& Zamparo, M., 2002. The explodingreflector concept for ground penetrating radar modeling. Annals of Geophysics 45: 473-478.

De Craen, M., Wang, L., Van Geet, M. \& Moors, H., 2004. Geochemistry of Boom Clay pore water at the Mol site Status 2004, Scientific Report BLG-990 04/MDC/P-48. SCK-CEN (Mol, Belgium).

Dutta, G. \& Schuster, G.T., 2014. Attenuation compensation for least-squares reverse time migration using the viscoacoustic-wave equation. Geophysics 79: S251-S262.

Elmore, C.W. \& Heald, A.M., 1969. Physics of Waves. McGraw-Hill Book Company (New York).

Gajewski, D. \& Tessmer, E., 2005. Reverse modelling for seismic event characterization. Geophysics Journal International 163: 276-284.

Hemerijckx, E., Carpentier, R., De Schrijver, P., Henriet, J.P. \& Heldens, Ph., 1983. Preliminary investigation of concretion horizons in a Tertiary clay layer in view of the construction of a pre metro tunnel under the river Scheldt at Antwerp (Belgium). Proceedings of the International Symposium on Engineering Geology and Underground Construction, Lisbon: pp. 53-70, http://lib.ugent.be/catalog/rug01:000199125.

Missiaen, T., Versteeg, W. \& Henriet, J.P., 2002. A new 3D seismic acquisition system for very high and ultra high resolution shallow water studies. First Break 20: 227-232.

Sena, A.R., Stoffa, P.L. \& Sen, M.K., 2006. Split-step Fourier migration of GPR data in lossy media. Geophysics 71: K77-K91.

Sun, J., Zhu, T. \& Fomel, S., 2015. Viscoacoustic modeling and imaging using the low-rank approximation. Geophysics 80: A103-A108.

Tukey, J.W., 1967. An introduction to the calculations of numerical spectrum analysis. Spectral Analysis of Time Series 25-46.
Vis, G.J. \& Verweij, J.M., 2014. Geological and geohydrological characterization of the Boom Clay and its overburden. OPERA-PU-TN0411 Scientific report. TN0 Geological Survey of the Netherlands (Utrecht).

Wang, Y. \& Guo, J., 2004. Seismic migration with inverse Q filtering. Geophysical Research Letter 31: L21608.

Zhang, Y., Zhang, P. \& Zhang, H., 2010. Compensating for visco-acoustic effects in reverse-time migration. SEG Technical Program Expanded Abstracts 31603164, http://dx.doi.org/10.1190/1.3513503.

Zhu, T., 2014. Time-reverse modeling of acoustic wave propagation in attenuating media. Geophysics Journal International 197(1): 483-494.

Zhu, T., Harris, J.M. \& Biondi, B., 2014. Q-compensated reverse-time migration. Geophysics 79: S77-S87.

\section{Appendix A: Phase velocity and attenuation and quality factors}

A plane-wave analysis of equation (3) in the homogeneous case, without the source term, implies $\left[\partial_{x}\left(\rho^{-1} \partial_{x} p\right)+\partial_{z}\left(\rho^{-1} \partial_{z} p\right)\right]=$ $\rho^{-1} \Delta p \rightarrow-\rho^{-1} k^{2} p, \partial_{t t} p \rightarrow-\omega^{2} p$ and $\partial_{t} p \rightarrow \mathrm{i} \omega p$, where $\Delta$ is the Laplacian, $k$ is the wavenumber, $\omega$ is the angular frequency and $\mathrm{i}=\sqrt{-1}$. With this substitution, we obtain

$$
c^{2} k^{2}=\omega^{2}-\gamma^{2}-2 i \gamma \omega .
$$

Then, the complex velocity is

$$
v=\frac{\omega}{k}=\frac{\omega c}{\omega-\mathrm{i} \gamma}
$$

(Carcione, 2015). From this quantity, the phase velocity and attenuation factor are

$$
v_{p}=\left[\operatorname{Re}\left(\frac{1}{v}\right)\right]^{-1}=c
$$

and

$$
\alpha=-\omega \operatorname{Im}\left(\frac{1}{v}\right)=\frac{\gamma}{c},
$$

respectively (e.g. Carcione, 2015) Both properties are frequency independent, meaning that there is no velocity dispersion.

On the other hand, defining the quality factor as the total energy density divided by the dissipated energy density (see equations (2.124), (3.131) and (3.132) of Carcione (2015)), we have

$$
Q=\frac{\operatorname{Re}^{2}(v)}{\operatorname{Im}\left(v^{2}\right)}=-\frac{\operatorname{Re}^{2}(k)}{\operatorname{Im}\left(k^{2}\right)}=\frac{\omega}{2 \gamma}
$$

The value of $\gamma$ can be obtained from the quality factor $Q_{0}$ at a given reference frequency $\omega_{0}=2 \pi f_{0}$, as

$$
\gamma=\frac{\omega_{0}}{2 Q_{0}} .
$$

\section{Appendix B: Finite-difference discretisation}

Assuming constant density, we discretise the Laplacian. Space and time are discretised as $x=i h, z=j h$ and $t=n d t$, where $h$ and $d t$ are the respective cell sizes. A suitable fourth-order 
accurate representation is

$$
\begin{aligned}
\Delta p_{i, j}= & \frac{1}{12 h^{2}}\left[-60 p_{i, j}+16\left(p_{i+1, j}+p_{i, j+1}+p_{i-1, j}+p_{i, j-1}\right)\right. \\
& \left.-\left(p_{i+2, j}+p_{i, j+2}+p_{i-2, j}+p_{i, j-2}\right)\right]
\end{aligned}
$$

(e.g., Abramowitz \& Stegun, 1964, p. 885), otherwise, the Fourier pseudospectral method can be used (e.g., Baysal et al., 1983; Carcione, 2015).

The time discretisation is

$$
\partial_{t t} p \rightarrow \frac{p_{i, j}^{n+1}-2 p_{i, j}^{n}+p_{i, j}^{n-1}}{d t^{2}}, \quad \partial_{t} p \rightarrow \frac{p_{i, j}^{n+1}-p_{i, j}^{n-1}}{2 d t} .
$$

Forward propagation is performed from equation (3) as

$$
\begin{aligned}
p_{i, j}^{n+1}= & \frac{1}{1+\epsilon}\left[\left(2-\epsilon^{2}\right) p_{i, j}^{n}-(1-\epsilon) p_{i, j}^{n-1}\right. \\
& \left.+c^{2} d t^{2} \Delta p_{i, j}+d t^{2} s^{\prime}\right],
\end{aligned}
$$

where $\varepsilon=\gamma d t$.

From equation (15), back-propagation is performed as

$$
p_{i, j}^{n-1}=\frac{1}{1-\epsilon}\left[\left(2-\epsilon^{2}\right) p_{i, j}^{n}-(1+\epsilon) p_{i, j}^{n+1}+c^{2} d t^{2} \Delta p_{i, j}\right] .
$$

Note that this equation is obtained from equation (15) if we consider a negative $Q_{0}$ (or negative $\varepsilon$ ), therefore backpropagation with equation (16) implies antidamping and recovery of the signal amplitude.

In the case of the exploding-reflector equation (4), the discretised equations are similar to equations (15) and (16), with the following substitution:

$$
c^{2} d t^{2} \Delta p_{i, j} \rightarrow c d t^{2} \Delta_{c} p_{i, j}, \quad \Delta_{c}=\partial_{x} c(x, z) \partial_{x}+\partial_{z} c(x, z) \partial_{z} p .
$$

In the migration process, the seismogram is a timedependent boundary condition in equation (16). The time step $d t$ is equal to the sample rate of the data. The seismic trace is applied at each receiver in reverse time and the propagation goes back in time until the origin time, where the best focusing occurs. The reverse modelling sums the energy of all receivers, enhancing the signal-to-noise ratio. The imaging condition is that of Gajewski \& Tessmer (2005), that is, the origin times of the events are given by the time where maximum focusing (maximum amplitude) occurs.

To prevent the growing of high-frequency noise, we use a low-pass Tukey filter in the wavenumber domain (Tukey, 1967; $\mathrm{Zhu}$, 2014). The cut-off wavenumber is calculated from the cutoff frequency based on the maximum phase velocity of the model. A suitable cut-off frequency is estimated by identifying the noise in the spectrum of the observed data.

\section{Appendix C: The Fresnel zone before and after migration}

The Fresnel zone is a measure of the horizontal resolution. Geobodies smaller than the Fresnel zone usually cannot be detected using seismic waves, but migration highly improves the resolution. Since the vertical resolution is $\lambda / 4$, where $\lambda$ is the wavelength, the horizontal resolution before migration, represented by the Fresnel radius, is

$$
R=\sqrt{\left(z^{2}+\frac{\lambda}{4}\right)^{2}-z^{2}} \approx \sqrt{\frac{\lambda z}{2}},
$$

where $z$ is the reflector depth and $\lambda=c / f_{p}$ (Elmore \& Heald, 1969).

Migration is a downward continuation of the seismic energy from the receivers to the reflectors such that the theoretical limit is obtained for $z=0$, that is, after migration the Fresnel radius is

$$
R=\frac{\lambda}{4}
$$

